\title{
Central Pain in Patients with Multiple sclerosis
}

\section{Feketová $\mathrm{S}^{1 *}$, Waczulíková $\mathrm{I}^{2}$, Valkovič $\mathrm{P}^{3}$ and Mareš $\mathrm{J}^{4}$}

${ }^{1}$ Department of Neurology, Faculty of Medicine, Comenius University and University Hospital, Bratislava, Slovak Republic ${ }^{2}$ Division of Biomedical Physics, Faculty of Mathematics, Physics, and Informatics, Comenius University, Bratislava, Slovak Republic ${ }^{3}$ Department of Neurology, Faculty of Medicine, Comenius University and University Hospital, Bratislava, Slovak Republic ${ }^{4}$ Department of Neurology, Faculty of Medicine, Palacky University and University Hospital Olomouc, Czech Republic

\begin{abstract}
Central pain $(\mathrm{CP})$ is little recognized and diagnosed type of pain in multiple sclerosis.

Objective: This second part of our work related to pain in multiple sclerosis is concentrated on occurrence of $\mathrm{CP}$ and defined its characteristics.

Methods: Questionnaires on pain were sent to 307 patients with definitive multiple sclerosis diagnose. Patients admitting to $\mathrm{CP}$ were examined with aim to diagnose CP. The dates were statistically processed.

Results: Out of 220 responders $92 \%$ reported pain during the course of their multiple sclerosis. CP was found in $57.72 \%$, including $40.91 \%$ with central neuropathic extremity and trunk pain (CNEP), $5.91 \%$ with trigeminal neuralgia (TN), and $33.18 \%$ with Lhermitte's sign (LS). In $28.8 \%$ of all patients, CP was an initial multiple sclerosis symptom. The prevalence of CP does not increase with age, disease duration, or the Expanded Disability Status Scale (EDSS). Lower extremities were the commonest location of CNEP (74.5\%) and burning was the commonest painful sensation. Three and more concurrent unpleasant painful sensations experienced $68.9 \%$ of patients, which were in $46.7 \%$ located in the lower extremities. Three and more concurrent CP locations (including TN and LS) were reported by $89 \%$ of patients. Number of locations in CP increases significantly with age and EDSS, in contrast to group with nociceptive and peripheral neuropathic pain $(n=113)$, where does not increase with age, EDSS and multiple sclerosis duration. The group with nociceptive and peripheral neuropathic pain featured significantly less patients with only one pain location $(p=0.0269)$ and only one pain quality. In contrast to the other group, In CP increases significantly the number of patients with increase the number of concurrent pain qualities $(p<0.0001)$.
\end{abstract}

Conclusion: $\mathrm{CP}$ is not only a frequent complaint among persons with multiple sclerosis, but is a distinctive type of pain requiring special attention and their identification remains still the major challenge.

Keywords: Multiple sclerosis; Pain, Prevalence; Neuropathic pain; Nociceptive pain

\section{Introduction}

Multiple sclerosis is a chronic, often disabling neurological disease with unpredictable and variable course. Among symptomultiple sclerosis, which vary from one person to another, pain is very common. Little recognized and diagnosed is central pain (CP). In practice, it is often overlooked, especially in the case of coexistence of several types of pain. CP is for the patient new feeling and different from pain as he knows before. Because of difficulties for many people with multiple sclerosis to find the right words to describe the painful sensations they experience ttherefore some $\mathrm{CP}$ sensations is being often unrecognised by clinician. Based on pathophysiological mechanism, the central pain is considered as neuropathic pain. According the mechanism-based classification of pain in multiple sclerosis distinguishes nine types of multiple sclerosis-related pain: trigeminal neuralgia and Lhermittes phenomenon (paroxysmal neuropathic pain due to ectopic impulse generation along primary afferent), on-going extremity pain (deafferentation pain secondary to lesion in the spino-thalamo-cortical pathways), painful tonic spasms and spasticity pain (mixed pains secondary to lesions in the central motor pathways but mediated by muscle nociceptors), pain associated with optic neuritis (nerve trunk pain originating from nervi nervorum), musculosceletal pains (nociceptive pain arising from postural abnormalities secondary to motor disorders), migraine (nociceptive pain favoured by predisponong factors or secondary to midbrain lesions) and treatment-induces pains. According to the International Association for the Study of Pain, CP is pain caused by a lesion or disease of the central somatosensory nervous system [1]. CP is commonly thought of as being excruciating pain with a bizarre character, covering small or large areas of body, mostly constant, but it may by intermittent or paroxysmal, varies in type and intensity among individuals [2]. No one pain quality is pathognomic for central pain, but concurrently more than one pain quality is occurring in multiple sclerosis patients [3] and the resulting pain is their combination. Almost any kind of lesion in the brain or spinal cord can cause $\mathrm{CP}$, which is associated with sensory disturbances, dominated by abnormalities in the sensibility to temperature and pain. $\mathrm{CP}$ is long-lasting, heavily tolerable, causing a great deal of suffering, while heat, pressure, as well as physical and mental overwork may increase the symptomultiple sclerosis. Often is extremely intense and intolerable during the night [4] with a significant impact on the quality of life (QOL) of the patients [5]. Central pain frequently develops at an interval of weeks or months after the occurrence of the lesion, does not seem to be dependent on etiology and may be the first symptom of multiple sclerosis, which may occur alone or together with other multiple sclerosis symptom-multiple sclerosis [6,7]. CP can be difficult to diagnose. The pain may be widespread and may seem unrelated to any injury or lesion. No single test is available to enable your doctor to diagnose CP. CP is very challenging to treat and may not respond to pharmacological agents routinely used for peripheral neuropathic pain [7]. As such, its management is troublesome. For all these reasons it is important to be familiar with the distinguishing features of central

*Corresponding author: Feketová, Department of Neurology, Faculty of Medicine Comenius University and University Hospital, Bratislava, Slovak Republic, Tel: +421903744144; Fax: +1-(718)-678-1018; E-mail: sona.feketova@gmail.com

Received June 14, 2017; Accepted August 16, 2017 ; Published August 23, 2017

Citation: Feketová S, Waczulíková I, Valkovič P, Mareš J (2017) Central Pain in Patients with Multiple sclerosis. J Mult Scler (Foster City) 4: 208. doi: 10.4172/23760389.1000208

Copyright: @ 2017 Feketová S, et al. This is an open-access article distributed under the terms of the Creative Commons Attribution License, which permits unrestricted use, distribution, and reproduction in any medium, provided the original author and source are credited 
neuropathic pain [7]. Results on prevalence studies of CP in multiple sclerosis are heterogenous, ranging from $14 \%$ to $64 \%$ among studies [4,6-11]. The most common type of central pain in multiple sclerosis is non-paroxysmal, continuous extremity pain (dysaesthesias or ongoing pain) of the arms, legs or trunk occurring in $14-29 \%$ of multiple sclerosis patients [4,6-11]. The common paroxysmal central pains in multiple sclerosis are trigeminal neuralgia with reported prevalence from 1.6 to $18 \%$ [5,6,11-14] and Lhermitte's sign with prevalence from 9 to $41 \%[1,11,13,15]$. Appropriate treatment of multiple sclerosis patients experiencing pain requires a thorough differentiation between nociceptive, peripheral neuropathic and central neuropathic pain. Each type of simultaneously occurring pain must be paid special attention to. Therefore, it is a great challenge to recognize and distinguish central pain from other types of pain.

\section{Patients and Methods}

The analysed group consisted of 307 patients (225 female and 82 male) aged 21 to 69 (with the average age of 37) with definitive multiple sclerosis in the patient register at the Department of Neurology of the University Hospital in Bratislava, Slovakia.

The patients were sent a questionnaire focusing on basic demographic characteristics (age, sex), disease specifics (onset of first multiple sclerosis symptom-multiple sclerosis, the year of diagnosis confirmation, form of the disease course, disability score) and pain.

Questions relating to pain were created on the basis of existing literature sources and our own clinical experiences. Responders were asked on onset of first painful sensations and if they had suffered from such types and qualities of pain as follows: headache, back or neck pain, trigeminal neuralgia, Lhermitte's sign, unpleasant painful sensations in upper legs, lower legs or other parts of the body (burning, cramping, stabbing, pressing, picking, stiffness, coldness) and secondary pain (due to urinary tract infection, spasticity, immobility, decubitus). Questionnaire replies received along with patient's informed consent were included in the study patient database and revised. All patients with unclear pain history were interviewed by telephone or examined at the outpatient clinic.

All patients with unpleasant painful sensations in extremities and trunk were examined with aim to diagnose central, peripheral neuropathic (peripheral neuropathy or radiculopathy) and nociceptive pain. Patients with dementia or psychiatric conditions were excluded from the study. Back pain, spasticity pain, headache and optic neuritis pain were considered as nociceptive pain in our study. Trigeminal neuralgia and Lhermitte's sign was considered as central pain. Non-painful paresthesias of central etiology and non-painful Lhermitte's sign were not considered as central pain. Painful tonic spasms were not included in the central pain. A control group without pain could not be formed for too low a number of painless multiple sclerosis patients participating in the study. In order to find out the same differences of patients with central pain we created a control group of multiple sclerosis patients with all others types of pain in multiple sclerosis. The group consisted of 113 patients (including 71 women) aged 25-53 years (mean 39 years).

The patients' data were collected and subsequently evaluated and interpreted using descriptive and inferential statistics. Categorical variables are presented as counts/percentages in tables and/or displayed graphically in the bar and pie charts. Continuous or interval-scaled variables were first checked for normality using graphical methods and the Shapiro-Wilk test. Differences in the proportions between responders and no-responders as well as between CNEP and nonCNEP groups were tested with binomial tests. Categorical variables grouped in two-way contingency tables were analysed using chi-square tests. In case of numerical calculability, exact tests were applied. Interval and continuous variables were tested using the Mann-Whitney test. To establish association between variables, we used corresponding bivariate or multivariate regression analysis methods. Chi-squared tests were used to determine the dependence between qualitative variables. The strength of the relationship was assessed using the GoodmanKruskal gamma correlation coefficient. Significance of expected or published predictors of the incidence of central pain was tested by bivariate and multivariate logistic regression and it is quoted with corresponding odds ratio (OR). All estimated statistics are presented along with the respective 95\% Confidence Interval (95\% CI). Our statistical significance reporting criteria for comparison between data sets was $\mathrm{p}<0.05$ (Figure 1). All analyses were conducted using Microsoft Office Excel 2003 (Microsoft Corporation) and StatsDirect 2.6 .6 (Stats Direct Ltd., Cheshire, UK) software.

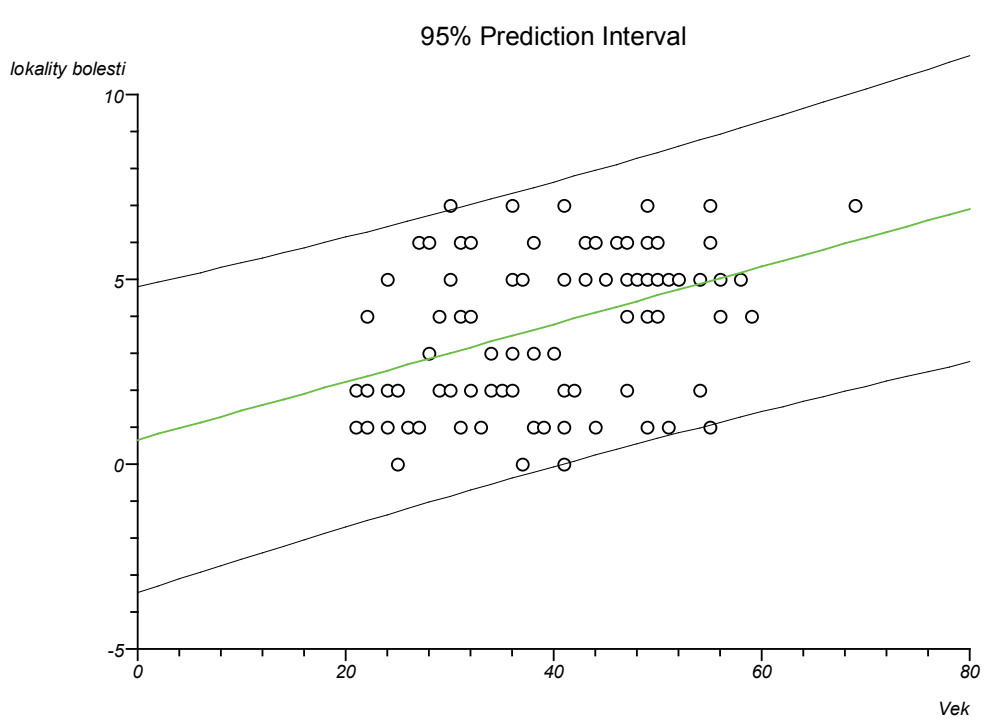

Figure 1: $95 \%$ prediction interval. 
Details from the study on prevalence of other types of pain than CP in multiple sclerosis was analyzed in detail in a separate study [17].

\section{Results}

Demographic data of the analyzed group of patients are presented in Table 1.

Questionnaires replies with informed consent were received from 220 patients (responders, i.e., the response rate was $71.7 \%$ (160 female and 60 male) aged 21 to 69 (with the average age of 39). Replies were not received from 87 patients (non-responders) (65 female and 22 male) aged 22 to 57 (with the average age of 36). The age, proportion of genders or disability score was not substantially different between responders and non-responders. Both groups had rather lower scores on the EDSS, which indicates a higher proportion of less disabled persons in our study. Both groups featured significantly more patients with relapsing-remitting multiple sclerosis $(\mathrm{p}<0.0001)$. The average disease duration (time since diagnosis) was similar in both groups (8 years for responders, 10 years for non-responders).

\section{Prevalence of pain in multiple sclerosis patients}

Pain during the course of disease reported 203 (92.3\%) of responders. Details from the study on prevalence at other than central pain in multiple sclerosis was analysed in detail in a separate study [17].

\section{Prevalence of central pain in multiple sclerosis patients}

$\mathrm{CP}$ was found to be common in 127 patients (57.72\%) of responders including those with central neuropathic extremity and trunk pain (CNEP) (90, 40.91\%), with trigeminal neuralgia (TN) $(13,5.91 \%)$ and those with Lhermitte's sign (LS) $(73,33.18 \%)$ (Table 2). All three types of $\mathrm{CP}$ occurred concurrently in four patients. $\mathrm{CP}$ occurred as an initial symptom of multiple sclerosis in $28.74 \%$ of responders (as CNEP in $28.74 \%$ and in $1.36 \%$ together with LS).

The prevalence of CP did not increase with age decades (the output from chi-square test: $\mathrm{p}=0.78$ ), and the presence of $\mathrm{CP}$ was not associated with age (the output from bivariate logistic regression: $\mathrm{p}=0.65 ; \mathrm{OR}=0.99,95 \% \mathrm{CI}$ (from 0.96 to 1.02 ).

The prevalence of $\mathrm{CP}$ was not associated with the EDSS score ( $\mathrm{p}=0.31$; OR $=0.89,95 \% \mathrm{CI}$ (from 0.71 to 1.12 ). Also regression analysis did not confirm EDSS as an independent predictor for manifestation of central pain.

The same finding was obtained for disease duration $(\mathrm{p}=0.68$; $\mathrm{OR}=0.98,95 \% \mathrm{CI}$ (from 0.93 to 1.04 ). Multivariable logistic regression did confirm non-significance of all three above mentioned predictors.

\section{Trigeminal neuralgia}

In our study, we found TN in $5.91 \%$ of patients; while in 2 patients (15\%) was bilateral. TN was the only painful sign of multiple sclerosis in one patient. Our results confirmed there is not an association between TN and EDSS $(p=0.51)$. The same finding was obtained for form of multiple sclerosis $(\mathrm{p}=0.7)$. The risk of this condition to occur was found to be equal in males and females $(\mathrm{OR}=0.452)$. We were not able to use the chi-squared test due to low counts; instead, we used the Fisher exact test. Our results confirmed there is no significant difference between males and females in the occurrence of trigeminal neuralgia $(\mathrm{p}=0.32)$, 95\% CI (from 0.06 to 1.90). The average age of persons with this condition was 41 years (the age range of 25-56), the average duration of trigeminal neuralgia was 13 years and the average time from diagnosis multiple sclerosis was 11 years. This condition was significantly more frequent in early-stage of disease duration and correlated with time since diagnosis multiple sclerosis $(\mathrm{p}=0.031 ; \mathrm{OR}=1.05,95 \% \mathrm{CI}$ (from 0.09 to 0.18 ).

\section{Lhermitte's sign}

The prevalence of painful LS was 73 patients (33.18\%), while in 36 responders ( $16.36 \%)$ LS was only painful symptom of multiple sclerosis and in three patients (1.36\%) onset of LS was in accordance with onset of the first symptomultiple sclerosis of SM, thus occurred at the disease onset. This condition was found equally frequent in males and females ( $\mathrm{p}=0.26$; OR $=0.686 ; 95 \%$ CI (from 0.35 to 1.30 ). No correlation was found between Lhermitte's sign and age, EDSS and disease duration.

\section{Central neuropathic extremity and trunk pain}

CNEP was identified in 90 patients (40.91\%), while in $28.74 \%$ of responders onset of CNEP was in accordance with onset of the first symptomultiple sclerosis of SM. CNEP was only painful symptom of multiple sclerosis in 47 patients (21.36\%) 10 patients (4.54\%) reported CNEP together with NT. Female were significantly more affected with CNEP than male $(\mathrm{p}<0.0001)$, 95\% CI (from 0.70 to 0.87$)$. OR $=0.537$, 95\% CI (from 0.27 to 1.03 ). 67 patients (74.5\%) reported unpleasant painful sensations located on lower extremities, 58 patients $(64.4 \%)$ on upper extremities, 39 patients $(43.3 \%)$ on a half of body and 31 patients $(34.4 \%)$ on a trunk (Table 4$)$. The commonest quality of pain was burning $(56.6 \%)$ (Table 3). 64 patients $(68.9 \%)$ reported three and more concurrent qualities of pain, which were in $46.7 \%$ of them located in the lower extremities. The commonest location of CNEP in $74.5 \%$ of the patients was lower extremities (Table 4). Three and more CP locations (including trigeminal neuralgia and Lhermittes sign were reported by $89 \%$ of the patients.

\begin{tabular}{|c|c|c|c|c|c|c|c|c|c|c|}
\hline & \multicolumn{2}{|c|}{ Patients } & \multicolumn{2}{|c|}{ Sex } & \multicolumn{2}{|c|}{ Age at examination } & \multicolumn{2}{|c|}{ EDSS } & \multicolumn{2}{|c|}{$\begin{array}{c}\text { Disease duration } \\
\text { (years) }\end{array}$} \\
\hline & number & $\%$ & W & M & range & mean & range & mean & range & mean \\
\hline Responders & 220 & 71 & 160 & 60 & $21-69$ & 39 & $2-6.5$ & 2.5 & 29-Jun & 8 \\
\hline Nonresponders & 87 & 29 & 65 & 22 & $22-58$ & 36 & 5-Jan & 2 & Jul-34 & 10 \\
\hline All patients & 307 & 100 & & & & & & & & \\
\hline
\end{tabular}

Table 1: Demographic characteristics.

\begin{tabular}{|c|c|c|c|c|c|c|}
\hline Central pain types & No. of patients & patients $\%$ & female & male & female $\%$ & male $\%$ \\
\hline Central neuropathic extremity and trunk pain & 90 & $\mathbf{4 0 . 9 1}$ & 72 & 18 & 20 \\
\hline Trigeminal neuralgia & 13 & $\mathbf{5 . 9 1}$ & 11 & 2 \\
\hline Lhermitte's sign & 73 & $\mathbf{3 3 , 1 8}$ & 57 & 17 \\
\hline
\end{tabular}

Table 2: Prevalence of central pain types in patients with MS. 
Citation: Feketová S, Waczulíková I, Valkovič P, Mareš J (2017) Central Pain in Patients with Multiple sclerosis. J Mult Scler (Foster City) 4: 208. doi: 10.4172/2376-0389.1000208

Page 4 of 6

\begin{tabular}{|c|c|c|c|c|c|c|c|c|}
\hline \multirow{2}{*}{ Unpleasant painful sensations } & \multicolumn{2}{|c|}{ Lower limbs } & \multicolumn{2}{|c|}{ Upper limbs } & \multicolumn{2}{|c|}{ A half of body } & \multicolumn{2}{|c|}{ Trunk } \\
\hline & $n$ & $\% T / \% C P$ & $n$ & $\% T / \% C P$ & $n$ & $\% T / \% C P$ & $n$ & $\% T / \% C P$ \\
\hline Burning & 52 & $23.6 / 55.9$ & 16 & $7.3 / 17.2$ & 16 & $7.3 / 17.2$ & 5 & $2.3 / 5.4$ \\
\hline Cramping & 36 & $16.4 / 38.7$ & 12 & $5.5 / 12.9$ & 9 & $4.1 / 9.7$ & 8 & $3.6 / 8.6$ \\
\hline Pressing Stiffness & 47 & $21.4 / 50.5$ & 34 & $15.5 . / 36.6$ & 27 & $12.3 / 29.0$ & 21 & $9.6 / 22.6$ \\
\hline Stabbing & 15 & $6.8 / 16.1$ & 14 & $6.4 / 15.1$ & 8 & $3.6 / 8.6$ & 8 & $3.6 / 8.6$ \\
\hline Painful coldness & 39 & $17.7 / 41.9$ & - & - & - & - & - & - \\
\hline Other types & 21 & $9.6 / 22.6$ & 25 & 27.78 & 10 & $4.6 / 10.8$ & 0 & $0 / 0$ \\
\hline
\end{tabular}

Table 3: Qualitative analysis of painful sensations occurring concurrently in patients with central neuropathic extremity and trunk pain.

\begin{tabular}{|c|c|c|c|c|c|c|c|c|}
\hline \multirow{2}{*}{$\begin{array}{c}\mathrm{n}: 90 \\
\text { sensations }\end{array}$} & \multicolumn{2}{|c|}{ Lower limbs } & \multicolumn{2}{|c|}{ Upper limbs } & \multicolumn{2}{|c|}{ A half of body } & \multicolumn{2}{|c|}{ Trunk } \\
\hline & $\mathrm{n}$ & $\%$ & $\mathrm{n}$ & $\%$ & $\mathrm{n}$ & $\%$ & $\mathrm{n}$ & $\%$ \\
\hline None & 23 & 25.6 & 32 & 35.6 & 51 & 56.7 & 59 & 65.6 \\
\hline One & 9 & 10 & 28 & 31.1 & 21 & 23.3 & 21 & 23.3 \\
\hline Two & 16 & 17.8 & 18 & 20 & 10 & 11.1 & 10 & 11.1 \\
\hline Three & 13 & 14.4 & 12 & 13.3 & 6 & 6.7 & 0 & 0 \\
\hline Four & 20 & 22.2 & 0 & 0 & 1 & 1.1 & 0 & 0 \\
\hline Five & 8 & 8.9 & 0 & 0 & 1 & 1.1 & 0 & 0 \\
\hline Six & 1 & 1.1 & 0 & 0 & 0 & 0 & 0 & 0 \\
\hline One & 9 & 10 & 28 & 31.1 & 21 & 23.3 & 21 & 23.3 \\
\hline Two & 16 & 17.8 & 18 & 20 & 10 & 11.1 & 10 & 11.1 \\
\hline Three and more & 42 & 46.7 & 12 & 13.3 & 8 & 8.9 & 0 & 0 \\
\hline
\end{tabular}

Table 4: Quantitative analysis of the number of concurrently occurring painful sensations in patients with central neuropathic extremity and trunk pain.

The number of locations of $\mathrm{CP}$ in the patient increased significantly with the age $(\mathrm{p}=0.0006$, correlation coefficient Gamma $=0.292$, 95\% CI (from 0.14 to 0.39 ).

The number of locations of CP increased significantly with EDSS ( $\mathrm{p}=0.0006$; correlation coefficient Gamma $=0.325,95 \%$ CI (from 0.16 to 0.52 ).

The number of locations with CP in the patient did not increase with duration of the disease (correlation coefficient Gamma $=0.153$; 95\% CI (from $-0,001$ to $0.27 ; \mathrm{p}=0.08$ ).

\section{Comparison of $\mathrm{CP}$ and other as $\mathrm{CP}$ (nociceptive and peripheral neurophatic-NCP)}

Groups of patients with CP and NCP were highly significantly different in terms of the number of pain qualities, which were in one patient present simultaneously (Table 5). While in the CP group the number of patients was growing with an increase of pain qualities occuring concurrently in one patient, in the NCP group with the increasing number of pain qualities the number of patients decreased (Fisher-Freeman-Halton exact test, $\mathrm{p}<0.0001$ ).

Groups of patients with $\mathrm{CP}$ and NCP were highly significantly different in terms of the number of pain sites, which were simultaneously present in one patient (Table 6). The number of patients with only one site of pain was significantly higher in the NCP group (Fisher-FreemanHalton exact test, $\mathrm{p}=0.0269$ ).

From our study was further revealed, that the number of NCP locations in the patients did not increase with the age (correlation coefficient Gamma=0.040, ( $\mathrm{p}=0.5724$ ), 95\% CI 95\% CI (from 0.14 to 0.39 ), the number of NCP locations in the patients did not increase with EDSS (correlation coefficient Gama $=0.090,(p=0.242)$, 95\% CI (from -0.03 to 0.19 ) and the number of locations with NCP in the patients did not increase with duration of the disease (correlation coefficient Gama $=0.021$, ( $\mathrm{p}=0.791), 95 \%$ CI (from -0.12 to 0.16 ).

\begin{tabular}{|c|c|c|c|c|c|}
\hline & $\mathbf{0 - 1}$ & $\mathbf{2 - 4}$ & $\mathbf{5 - 8}$ & $\mathbf{9}$ and more & all \\
\hline $\mathbf{( n = 9 0 )}$ & & & & & \\
\hline $\mathbf{C P}(\mathbf{n})$ & 15 & 23 & 24 & 28 & 90 \\
\hline $\mathbf{C P}(\%)$ & $16.67 \%$ & $25.56 \%$ & $26.67 \%$ & $31.11 \%$ & $100 \%$ \\
\hline$(\mathrm{n}=113)$ & & & & & \\
\hline $\mathbf{N C P}(\mathbf{n})$ & 18 & 52 & 33 & 10 & 113 \\
\hline NCP $(\%)$ & $13.85 \%$ & $49.35 \%$ & $29.11 \%$ & $7.69 \%$ & $100 \%$ \\
\hline
\end{tabular}

Table 5: Comparison of the number of pain qualities in MS patients occurring concurrently in CP or in NCP.

\begin{tabular}{|c|c|c|c|}
\hline & $\mathbf{H : 0}$ & $\mathbf{H : 1}$ & $\mathbf{H : 2}$ and more \\
\hline $\mathbf{C P}(\mathbf{n})$ & 3 & 15 & 72 \\
\hline $\mathbf{C P}(\mathbf{\%})$ & $3.33 \%$ & $16.67 \%$ & $80 \%$ \\
\hline $\mathbf{N C P}(\mathbf{n})$ & 12 & 9 & 109 \\
\hline $\mathbf{N C P}(\%)$ & $9.23 \%$ & $6.92 \%$ & $83.85 \%$ \\
\hline
\end{tabular}

n: number, $\mathrm{H}$ : number of pain locations, CNEP: Central Extremity and Trunk Pain, NCNEP: Other as Central Extremity and Trunk Pain (Non-Central Extremity and Trunk Pain)

Table 6: Comparison of the number of pain sites occurring concurrently in patients with CP or NCP.

\section{Discussion}

\section{Representativeness of sample}

Based on the assumption that in our analysis of 200 patients (approx. $5 \%$ of the multiple sclerosis population in Slovakia), there is an even distribution of characteristics within the population, our sample might be considered as representative. Regarding proportional representation by age our set of patients is consistent with epidemiological data on multiple sclerosis [18]. Regarding proportional representation by gender, it correlates with epidemiological data concerning multiple sclerosis $[15,19]$. Finally, from the viewpoint of proportional representation of patients stratified by multiple sclerosis forms, we found our sample to be just as consistent with epidemiological data [18]. 


\section{Prevalence of central pain}

Concerning to the effort to specify the prevalence of central pain in patients with multiple sclerosis, there was done an estimation of the necessary number of the patients with multiple sclerosis. The estimated size of the group in terms of characteristics obtaining we set up in accordance to the population incidence of clinically confirmed central pain and the difference was considered as an acceptable deviation of $\pm 20 \%$ from percentage of prevalence. Estimation of prevalence rate we did on the base of published articles and the level of reliability of the estimation we set up to $95 \%$. Result for the needed selection from patients' population with SM (8000) and for a published middle-sized prevalence is 218 . This corresponds with our group of patients.

Our research found central pain in more than half of the patients (57.72\%), which is comparable with other published studies [20-22]. Compared to other findings $[4,6,9,11]$ the prevalence of central pain in our study turned out to be higher. This might have been caused by geographical and demographic factors of the prevalence of multiple sclerosis itself, differing patients samples, study design and by our opinion on recognition this very distinctive type of pain by patients, because of difficulties to find the right words to describe this new painful feeling and also insufficient attention to obtain reliable information about painful sensations from the patients and thus to diagnose pain of central neuropathic origin by physician.

Our results could be influenced by inclusion of some types of neuropathic pain, which were not included in some previous studies. According to literature sources most of the studies reported as central pain only neuropathic extremity pain alone or in combination with trigeminal neuralgia $[4,6,13,20]$ Only in the, study by Foley, where investigators used pooled prevalence estimates, explored heterogeneity using meta-regression, and analysed prevalence during the disease course using both estimates at disease milestones and longitudinal studies, was in central neuropathic pain included neuropathic extremity pain (7.1\%-52.8\%), Lhermitte's sign (9.7\%-25.0\%) and trigeminal neuralgia $(2.0 \%-6.0 \%)$ with pooled prevalence of CNEP $26 \%$, LS $16 \%$ and TN 3.8\%. The prevalence of central pain, according to our results, does not increase with patient age, disease duration or EDSS [4]. Thus it appears that neither the increasing age of the patient, EDSS score nor duration of multiple sclerosis increases the risk for development of CP. This conclusion is in accordance with my own clinical experiences partly in accordance with study by Österberg et al. where it was found out, that $\mathrm{CP}$ prevalence does not increase with higher degree of disability and out of the results of their study was concluded, that it appears that neither increasing age nor increasing duration of disease increase the risk for developing CP. These interesting results contradict previous findings on the prevalence of overall pain in patients with multiple sclerosis, where it was found that the pain prevalence increased with age $[4,8,10,13]$ disease duration $[13,23]$ as well as increasing disability $[10,13,23]$. As in studies on prevalence of overall pain in multiple sclerosis except central neuropathic extremity pain and trigeminal neuralgia, there were nociceptive and peripheral neuropathic pain included and according Osterberg finding, that prevalence of peripheral neuropathic pain in multiple sclerosis is very low, thus it appears that responsible for different results of the studies could be nociceptive pain [6]. Based on the above findings, it can be assumed that the increasing age, increasing EDSS and duration of the disease in patients with multiple sclerosis increase the risk for developing nociceptive, but not for central neuropathic, especially central neuropathic extremity pain. Further studies are needed to confirm this theory.

\section{Characteristics of types of central pain}

Trigeminal neuralgia: After revision all our patients with TN confirmed criteria according Cruccu et al. Our results showed TN to be present in $5.91 \%$ of multiple sclerosis patients, which is much higher than in general population (from $0.1 \%$ to $0.7 \%$ ) [24]. This is consistent with published data reporting the prevalence range $\mathrm{TN}$ in multiple sclerosis from $1.6 \%$ to $18 \%[3,8,19,11,24]$. The age of onset of multiple sclerosis- associated TN in patients has been noted to be about 10 years younger than in patients without multiple sclerosis [25-27]. In our study the average age of persons with this condition was 41 years (the age range of 25-56), while average duration of trigeminal neuralgia was 13 years and average time from diagnosis multiple sclerosis was 11 years, which pointed to onset in the early period of multiple sclerosis and at young age. These results are in accordance with published studies about early age of onset of multiple sclerosis-associated TN than in patients without multiple sclerosis [24-27] and have more years to develop recurrence, also are more likely to be medically intractable because their multiple sclerosis symptomultiple sclerosis are often worsened by the anti-seizure medicines used to treat TN [28], thus suffer much.

Lhermitte's sign: According to the Multiple sclerosis Foundation approximately 38 percent of multiple sclerosis patients will experience Lhermitte's sign at one time or another. In literature, the data on prevalence of this disorder vary from $2 \%$ to $41 \%$ [11,13,29-31]. The possible explanation for differences in prevalence of Lhermitte's sign may be the fact, that there is some multiple sclerosis patients for which an electric feeling passing down the back to the legs on flexing the neck (the common form of Lhermitte's sign) could be painless. One-quarter of patients with Lhermitte's sign had never told their physicians of these symptom-multiple scleroses (Al-Arai). In literature, there are few studies which concentrate their attention to LS as painful symptom in multiple sclerosis. Our questionnaire of pain was focusing on description of perceiving painful sensations, thus in our study LS was presented as painful. The prevalence of $34.7 \%$ is within the published range. No correlation was found between Lhermitte's sign and age, EDSS and disease duration. This is according with study by Beckmann. Furthermore we found out, that in a half of patients with LS, it was only painful symptom of SM and in $1.36 \%$ of the patients LS occurred at the disease onset, which isn't much different from 3\% published by Paty and Poser. Because of limitation in current knowledge regarding LS in multiple sclerosis, there is a need for future studies that would increase understanding in this area.

Central neuropathic extremity pain: The most common painful symptomultiple sclerosis of neuropathic pain in our population and often one of the first symptomultiple sclerosis of pain perceived at the beginning of multiple sclerosis is pain felt in the extremities [9]. Most of these painful symptomultiple sclerosis are of central neuropathic origin. In our series $40.91 \%$ of patients experienced central neuropathic extremity and trunk pain, which is in the range of published studies (7.1\%-52.8\%) examined by Foley [4].

The prevalence of CNEP as part of the onset symptomatology of multiple sclerosis was not surprising very high (28.74\% of responders), because this type of pain is a directly consequence of the multiple sclerosis lesion. In accordance with literature sources [5,6,22,32] the most commonly perceived quality of pain in our study were burning and painful cold. Compared with the general population experiencing chronic neuropathic pain, multiple sclerosis patients were more likely to use the pain adjectives of painful cold, tingling, itching, and numbness [33]. Such descriptors of neuropathic pain in multiple sclerosis patients could be related to central mechanisms, whereas in the general population, neuropathic pain is mostly due to peripheral mechanisms [22]. 
The significant interindividual differences have been found in quality of pain perceiving by the patients, who often indicate more than one type of pain [6], and the resulting pain is their combination.

So pathognomonic for central pain is not certain quality of pain, but the combination of different qualities of pain [34]. In the present study more than two third of CNEP patients experienced three and more concurrent unpleasant painful sensations on lower extremities and almost two third on upper extremities, which is in line with the study conducted by Leijon et al. [18] for central pain after stroke (stroke) and study by Österberg et al. [19] and also Moisset [20] for central pain in multiple sclerosis.

The most important finding was that among our patients with $\mathrm{CP}$, $89 \%$ were presented with three and more painful sites at the same time.

The number of locations in CP patients increases significantly with patient age, increasing degree of the EDSS and a hint of the trend is the duration of SM. The number of locations in NCP patients did not increase with the age, EDSS and duration of the disease. Such a correlation could not be found in many other studies.

\section{Conclusion}

Our study confirms that central pain is a frequent and unique complaint among persons with multiple sclerosis for which is patognomic combinations of different concurrent pain qualities, no one quality of pain, which are experiencing in multiple sites at the same time. The prevalence of $\mathrm{CP}$ is high and in nearly $30 \%$ of the patients are present in the early stages of the disease together with other symptommultiple sclerosis, while during the disease the number of painful sites and the qualities of pain experienced by patients is raising. On the basis of these facts is extremely important to pay great attention to this type of pain not only by researchers but by doctors too.

This finding, along with other results of our study highlights the special status of central pain in SM. Necessary but further studies to improve our knowledge and assess the significance of central pain and its place within the complexity of the patients with multiple sclerosis.

\section{References}

1. Jensen TS, Baron R, Haanpää M, Kalso E, Loeser JD, et al. (2011) A new definition of neuropathic pain. Pain 152: 2204-2205.

2. Boivie J (2006) Central pain. Wall and Melzack's Textbook of pain. Elsevier Inc., London 1057-1075.

3. Putzki N, Pfriem A, Limmroth V, Yaldizli O, Tettenborn B, et al (2009) Prevalence of migraine, tension-type headache and trigeminal neuralgia in multiple sclerosis. Eur J Neurol 16: 262-267.

4. Moulin DE, Foley KM, Ebers GC (1988) Pain syndromes in multiple sclerosis. Neurology 38: 1830-1834.

5. Svendsen KB, Jensen TS, Overvad K, Hansen HJ, Koch-Henriksen N, et al. (2003) Pain in patients with multiple sclerosis: A population-based study. Arch Neurol 60: 1089-1094.

6. Österberg A, Boivie J, Thuomas KA (2005) Central pain in multiple sclerosisprevalence and clinical characteristic. Eur J Pain 9: 531-542.

7. Watson JC, Sandroni P (2016) Central neuropathic pain syndromes. Mayo Clin Proc 91: 372-385.

8. Clifford DB, Trotter JL (1984) Pain in multiple sclerosis. Arch Neurol 41: 12701272.

9. Vermote R, Ketelaer P, Carton H (1986) Pain in multiple sclerosis patients. A prospective study using Mc Gill Pain Questionnaire. Clin Neurol Neurosurg 88: 87-93
10. Stenager E, Knudsen L, Jensen K (1991) Acute and chronic pain syndromes in multiple sclerosis. Acta Neurol Scand 84: 197-200.

11. Foley PL, Vesterinen HM, Laird BJ, Sena ES, Colvin LA, et al. (2013) Prevalence and natural history of pain in adults with multiple sclerosis: Systematic review and meta-analysis. Pain 154: 632-642.

12. Hooge JP, Redekop WK (1995) Trigeminal neuralgia in multiple sclerosis Neurol 45: 1294-1296.

13. Solaro C, Trabucco E, Messmer Uccelli M (2013) Pain and multiple sclerosis: Pathophysiology and treatment. Curr Neurol Neurosci Rep 13: 320.

14. Putzki N, Pfriem A, Limmroth V, Yaldizli O, Tettenborn B, et al (2009) Prevalence of migraine, tension-type headache and trigeminal neuralgia in multiple sclerosis. Eur Neurol 16: 262-267.

15. Sadovnick AD, Ebers GC (1993) Epidemiology of multiple sclerosis: A critical overview. Can J Neurol Sci 20: 17-29.

16. Jensen TS, Baron R, Haanpää M, Kalso E, Loeser JD, et al. (2011) A new definition of neuropathic pain. Pain 152: 2204-2205.

17. Feketova S, Waczulikova I, Kukumberg P, Mares J (2016) Pain in multiple sclerosis: Prevalence and characteristics of various pain conditions. J Mult Scler (Foster City) 3: 187.

18. Compston A, Confravreux C (2006) The distribution of multiple sclerosis McAlpine's Multi Scler 4: 71-112.

19. Alonso A, Hernán MA (2008) Temporal trends in the incidence of multiple sclerosis: A systematic review. Neurol 71: 129-135.

20. Khan F, Pallant J (2007) Chronic pain in multiple sclerosis: Prevalence, characteristics and impact on quality of life in an Australian community cohort. J Pain 8: 614-623.

21. Seixas D, Galhardo V, Sá MJ, Guimarães J, Lima D (2009) Pain in multiple sclerosis: Characterization of a Portuguese population of 85 patients. Acta Med Port 22: 233-240.

22. Moisset X, Ouchchane L, Guy N, Bayle DJ, Dallel R, et al. (2013) Migraine headaches and pain with neuropathic characteristics: Comorbid conditions in patients with multiple sclerosis. Pain 1542: 691-2699.

23. Grau-López L, Sierra S, Martínez-Cáceres E, Ramo-Tello C (2011) Analysis of the pain in multiple sclerosis patients. Neurologia 26: 208-213.

24. O'Connor AB, Schwid SR, Herrmann DN, Markman JD, Dworkin JH, et al (2008) Pain associated with multiple sclerosis: Systematic review and proposed classification. Pain 137: 96-111.

25. De Simone R, Marano E, Brescia Morra E, Ranieri A, Ripa P, et al (2005) A clinical comparison of trigeminal neuralgia pain in patients with and without underlying multiple sclerosis. Neurol Sci 26: 150-151.

26. Jensen TS, Baron R, Haanpää M, Kalso E, Loeser JD, et al. (2011) A new definition of neuropathic pain. Pain 152: 2204-2205.

27. Brisman R (1987) Trigeminal neuralgia and multiple sclerosis. Arch Neurol 44 379-381.

28. Brisman, R (2013) Treatment of trigeminal neuralgia associated with multiple sclerosis. World Neurosurg

29. Pöllmann W, Feneberg W (2008) Current management of pain associated with multiple sclerosis. CNS Drugs 22: 291-324.

30. Sandyk R, Dann LC (1995) Resolution of Lhermitte's sign in multiple sclerosis by treatment with weak electromagnetic fields. Int J Neurosci 81: 215-224.

31. Al-Araji AH, Oger J (2005) Reappraisal of Lhermitte's sign in multiple sclerosis Mult Scler 11: 398-402

32. Svendsen KB, Jensen TS, Hansen HJ, Bach FW (2005) Sensory function and quality of life in patients with multiple sclerosis and pain. Pain 114: 473-481.

33. Bouhassira D, Lantéri-Minet M, Attal N, Laurent B, Touboul C (2008) Prevalence of chronic pain with neuropathic characteristics in the general population. Pain 136: $380-387$.

34. Leijon G, Boivie J (1989) Central post-stroke pain--the effect of high and low frequency TENS. Pain 38: 187-191.

Citation: Feketová S, Waczulíková I, Valkovič P, Mareš J (2017) Central Pain in Patients with Multiple sclerosis. J Mult Scler (Foster City) 4: 208. doi: 10.4172/2376-0389.1000208 